\title{
Different consecutive training protocols to design an intervention program for overweight youth: a controlled study
}

This article was published in the following Dove Press journal:

Diabetes, Metabolic Syndrome and Obesity:Targets and Therapy

16 January 2017

Number of times this article has been viewed

\author{
Giovanni Fiorilli' \\ Enzo Iuliano' \\ Giovanna Aquino' \\ Emidio Campanella' \\ Despina Tsopani ${ }^{2}$ \\ Alfonso Di Costanzo' \\ Giuseppe Calcagno' \\ Alessandra di Cagno 3 \\ 'Department of Medicine and Health \\ Sciences, University of Molise, \\ Campobasso, Italy; ${ }^{2}$ Department of \\ Motor and Health Sciences, National \\ Kapodistrian University of Athens, \\ Athens, Greece; ${ }^{3}$ Department of \\ Motor, Human and Health Sciences, \\ University of Rome "Foro Italico", \\ Rome, Italy
}

Objective: To find the optimal exercise program to be recommended in reducing adiposity and promoting long-term physical activity adherence in a sample of overweight adolescents.

Methods: Forty-five overweight adolescents were randomly divided into three exercise groups, to perform two phases of physical activity as follows: in the first phase, the first group performed a 16-week moderate-intensity resistance training (RT), the second group performed a 16-week high-intensity RT, and the third group performed a 16-week aerobic training (AT); in the second phase, all groups performed a 6-week AT. Anthropometric body composition and fitness measures were considered as outcome measures.

Results: After the second protocol, both RT groups showed a significant improvement in percentage of fat mass $\left(F_{2,76}=5.843 ; p=0.004 ; \eta^{2}=0.133\right)$ and free fat mass $\left(F_{2,76}=6.254 ; p=\right.$ $\left.0.003 ; \eta^{2}=0.141\right)$, and in fitness tests $(p<0.01)$. The $\mathrm{VO}_{2} \max$ values of the RT groups were significantly higher than those of the AT group $\left(F_{2,38}=4.264 ; p=0.021 ; \eta^{2}=0.183\right)$. The rate of adherence to exercise was an average of $94 \%$ in both RT groups, whereas in the AT group, it was $83 \%$. During the 12 -week post-intervention follow-up, the number of participants who continued to perform physical activities was significantly higher in both the RT groups than in the AT group $(p<0.05)$.

Conclusion: The present study provides preliminary evidence that moderate-to-intense RT, followed by AT, can be an effective treatment for overweight adolescents, and the positive effects are maintained even after 12 weeks of follow-up.

Keywords: exercise, obesity, adolescents, adherence, resistance training

\section{Introduction}

In the last decades, resistance training (RT) has been recommended for children and adolescents which is to be applied with appropriate techniques and correctly supervised. ${ }^{1}$ Indeed, the risks of injuries, due to the physical activity proposed to overweight adolescents, can be minimized by reducing training loads, using age-adequate equipment and ensuring adequate recovery between the training sessions. ${ }^{2}$ Low-intensity RT programs do not guarantee an adequate training stimulus and could lead to difficulty in differentiating training adaptation from normal growth. ${ }^{3}$ High-intensity progressive RT leads to reduction in adiposity and metabolic risk in normal-weight and overweight children, ${ }^{4}$ increasing the muscle mass, which has been associated with improved insulin sensitivity. ${ }^{5}$ Considering that continuity of physical activity is a necessary condition for long-term maintenance of weight loss, it is essential that the dose, mode and intensity should be appropriate and enjoyable to this target group. ${ }^{6,7}$ Although overweight youth have traditionally been encouraged to participate in aerobic activities, the excess of
Correspondence: Giuseppe Calcagno Department of Medicine and Health Sciences, University of Molise, Via de Sanctis, I 86I00 Campobasso, Italy Tel +39 874404402

Email giuseppe.calcagno@unimol.it 
body weight increases the risk of musculoskeletal injuries, decreasing self-confidence and enjoyment. ${ }^{8}$ Aerobic exercise is usually avoided by overweight and obese adolescents to avoid being further ridiculed by their peers, whereas strength training provides an opportunity for them to experience success and feel good about their performance in which they often are better than their underweight peers. ${ }^{9}$ Overweight adolescents show better adherence to RT, typically characterized by short period of loading exercises, with rest periods between sets and repetitions. An RT-conditioning program improves the socialization and mental discipline of participants who perceive fast self-improvement. ${ }^{10}$ In fact, obesity is associated with low self-esteem, depressive mood and impairment in emotional well-being. ${ }^{11,12}$ Consequently, the typology and methodology of training should respect the individual needs, goals and abilities in order to optimize gains, to prevent boredom and to promote exercise adherence. ${ }^{13}$

The aim of this study was to find the optimal exercise program to be recommended for overweight adolescents to reduce adiposity, improve free fat mass (FFM) and to promote long-term physical activity adherence in order to maintain the benefits of physical activity. It was hypothesized that both moderate- and high-intensity programs of RT may provide strength and metabolic benefits to this population and may positively prepare them to the successive phase of aerobic program (AP). Moreover, it was expected that the RT, promoting satisfaction with RT-related outcomes, could lead the participants to continue the following AP, important in terms of weight reduction.

\section{Methods}

This was an intervention study with three parallel groups. The study protocol was divided into two phases: in the first (phase I), lasting 16 weeks, the first of the two RT groups performed a moderate-intensity RT and the second group a high-intensity RT, and the aerobic training (AT) group performed AP; in the second phase (phase II), lasting 6 weeks, all the three groups performed AP. At the end of the intervention, a 12-week post-intervention follow-up was made. Anthropometric measures, body composition and fitness parameters were assessed at baseline, after phase I and phase II and after follow-up period (Figure 1).

Forty-five overweight adolescents of both genders were enrolled in the study, but only 41 completed all phases of the study. The eligibility requirements were age $\geq 12$ and $\leq 15$ years, sedentary lifestyle, percentage of body fat $\geq 25 \%$ for male and $\geq 30 \%$ for female and body mass index (BMI) $>$ 85 th percentile, in comparison with the representative data of adolescents of the same age, ${ }^{14,15}$ and a valid medical certificate. The exclusion criteria were: recent history of injury, presence of metabolic syndrome, cardiovascular diseases and low adherence to the training sessions (absences $>20 \%$ ). The flowchart of the study is reported in Figure 2.

The Ethics Committee of University of Molise reviewed and approved this study. A written informed consent was obtained from all participants and their parents before the participants were recruited for the study, in accordance with the principles outlined in the Declaration of Helsinki. All data obtained for the study were anonymized.

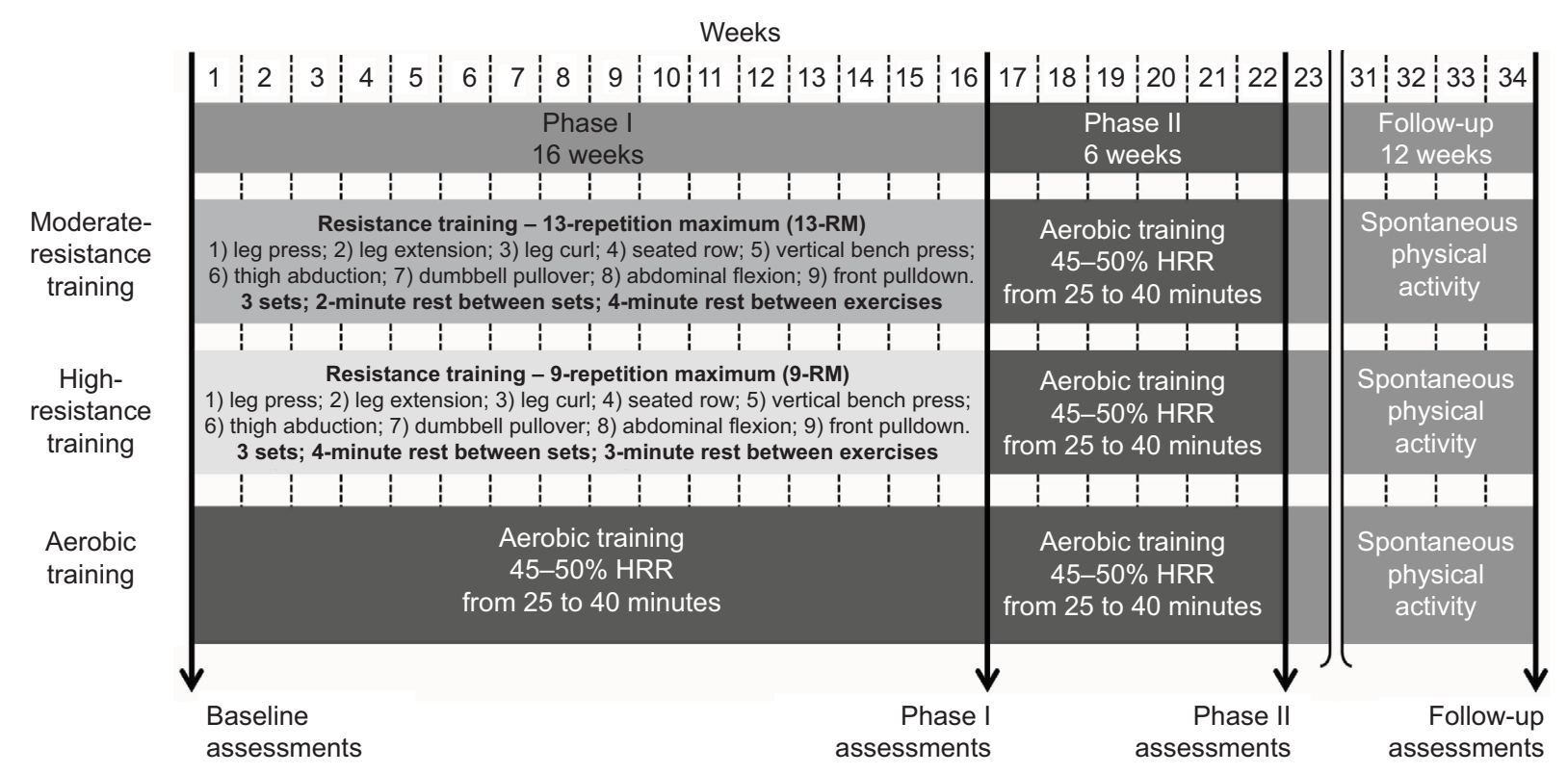

Figure I Study design

Abbreviation: HRR, heart rate reserve. 


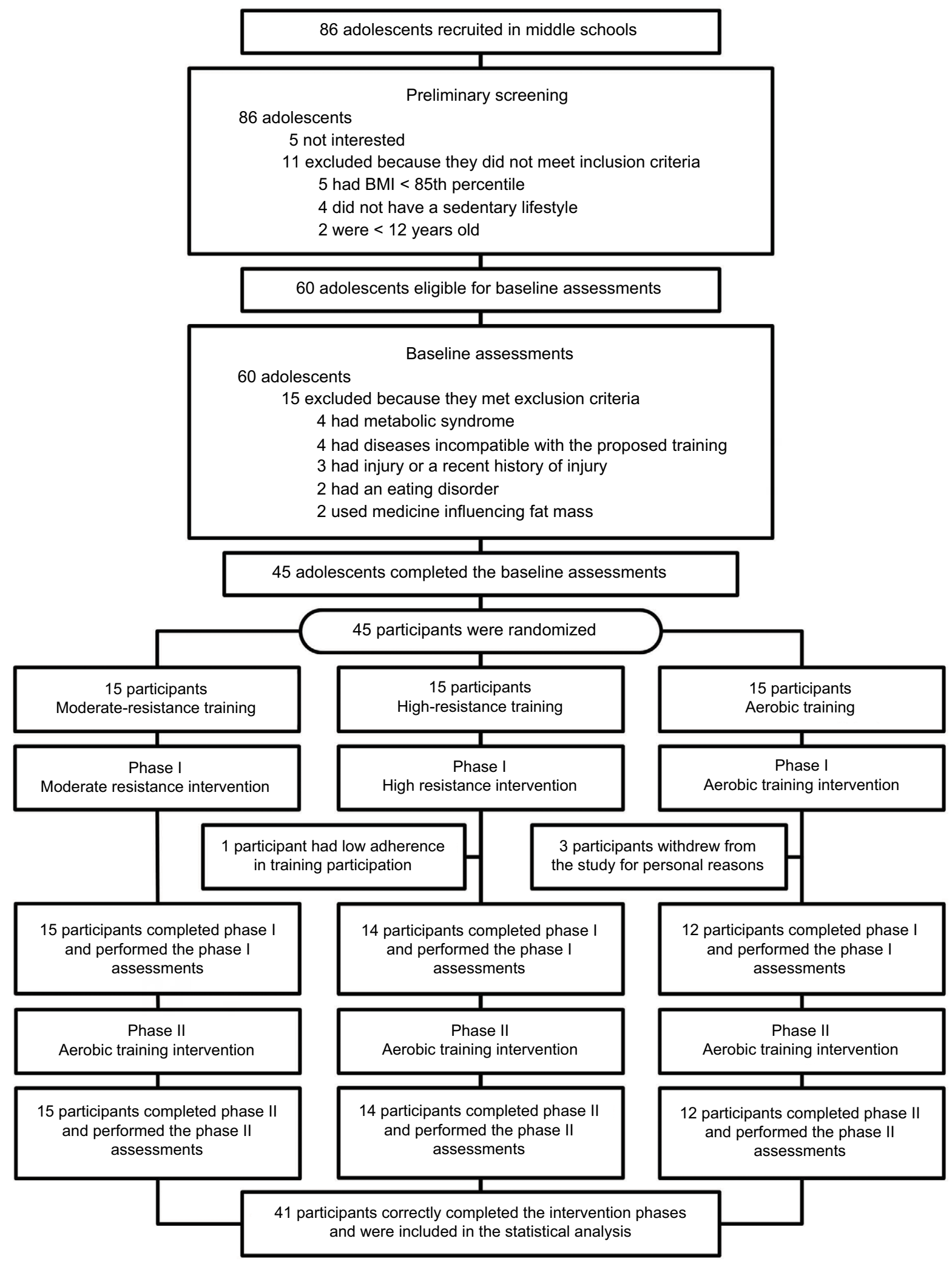

Figure 2 Flowchart of the intervention program.

Abbreviation: BMI, body mass index.

After the baseline assessment, the participants were randomly divided into three groups: moderate-resistance training (MRT), high-resistance training (HRT) and AT.
They were randomized into groups of six, with two subjects randomly assigned to each group according to a sequence of computer-generated random numbers (SPSS version 20.0; 
IBM, Armonk, NY, USA). This procedure was designed to obtain groups of equal sizes and to avoid selection bias.

During phase I, the MRT group performed the RT at isotonic machines with an intensity of 13-repetition maximum (13-RM), three sessions per week. The HRT performed the same exercises protocol with the same modality of MRT group, but with higher intensity (9-repetition maximum, 9-RM). The number of sets and repetitions was not modified during phase I, but the loads were modified in accordance with the strength improvement reached by the participants in order to assure an intensity of 9-RM and 13-RM. At baseline, the 9-RM and the 13-RM intensities, respectively used in HRT and MRT, were assessed during the familiarization sessions, and in the following weeks, the 9-RM and the 13-RM intensities were assessed every 3 weeks during the session of training. For the interpretation of the strength improvement, 1-RM was also indirectly estimated using the Brzycki submaximal method. ${ }^{16}$ This indirect evaluation was used in order to ensure the safety of the participants.

The AT group performed AP, three sessions per week, at $45-50 \%$ of heart rate reserve, using cycle-ergometers and treadmills. The duration of each session was progressively increased from 25 minutes up to 40 minutes, adding 5 minutes every following session, with 10 minutes of warm-up and cool-down. During phase II, all the three groups performed AP with the same modalities of the AT group in phase I.

The physical activity was supervised by three professional personal trainers appointed for each group (the ratio of personal trainer and participants was at least 1:5) in order to assure the safety of the participants, the correct execution of the exercises and the adherence to the protocols. The heart rate was monitored using Polar Team 2 system (Polar Electro Oy, Kempele, Finland). All the participants performed two preliminary sessions to get familiarized with the correct use of isotonic machines and ergometers and to assess the RT loads (13-RM and 9-RM).

At the end of the intervention, the participants were exhorted to continue the physical activity, and after 12 weeks, they were reassessed (follow-up). A questionnaire was administered in order to investigate if they had an active daily life and continued their physical activity program. Based on the results of the questionnaire, the participants were classified into two categories: positive continuer (POS-CO) consisting of the participants who continued the physical activities, and negative continuer (NEG-CO) consisting of those who did did not continue the physical activity properly, after the end of the intervention. The follow-up assessment aimed to evaluate if the three groups had long-term benefits on anthropometric measures and body composition in comparison with their starting condition (baseline evaluation) and the condition achieved at the end of the intervention (phase II evaluation).

\section{Anthropometric and body composition evaluation}

Height, weight, BMI, waist circumference and waist-hip ratio were measured in order to evaluate macroscopic changes in the body measures. Internationally acceptable cut-off points for waist circumference were used in this study. ${ }^{17}$ Body composition was assessed using skinfold measurements. The skinfolds were measured on the right side of the body and recorded to the nearest $0.1 \mathrm{~mm}$. The measurements were taken in triplicate by the same investigator, and the average of the three measurements was used in the data analysis.

A skinfold caliper was used to measure biceps, triceps and subscapular and supra-iliac skinfolds. The measures obtained (in millimeters) were included in the Johnston et $\mathrm{al}^{18}$ equation in order to obtain the body density of the participants. The body density was therefore used to calculate their percentage of fat mass (\%FM) using the equation of Weststrate and Deurenberg, ${ }^{19}$ specific for adolescent population. A study of Rodríguez et $\mathrm{al}^{20}$ calculated that the predicted $\% \mathrm{FM}$ assessed with this procedure has an absolute error of $5.92 \%$ in female and $3.90 \%$ in male subjects compared with the measurement obtained with the dual-energy X-ray absorptiometry. The FFM (in kilograms) of the participants was also calculated in order to evaluate modification in their lean body mass.

\section{Physical assessment}

Physical assessment was carried out using the specific tests of the Eurofit Test Battery for flexibility, ${ }^{21}$ agility, resistance and balance, and the Queens College Step Test for aerobic fitness. ${ }^{22}$ All the tests were performed between 9 and $12 \mathrm{am}$. The Queens College Step Test is used for estimating maximal oxygen uptake. The protocol consisted of stepping up and down on a platform of $41.3 \mathrm{~cm}$, at a rate of 22 and 24 steps per minute (for females and males, respectively), for 3 minutes. The participants stopped the test after the third minute, and their heart rate was measured as beats per minute (bpm) after 20 seconds of recovery. The heart rate was assessed using a Polar A300 heart rate monitor, with $\mathrm{H} 7$ heart rate sensor. The estimation of maximum oxygen consumption $\left(\mathrm{VO}_{2} \max \right)$ was made using one of the two following equations according to the gender: for males, $\mathrm{VO}_{2} \max \left(\mathrm{mL} \cdot \mathrm{kg}^{-1} \cdot \mathrm{min}^{-1}\right)=111.33-$ $(0.42 \times \mathrm{bpm})$; for females, $\mathrm{VO}_{2} \max \left(\mathrm{mL} \cdot \mathrm{kg}^{-1} \cdot \mathrm{min}^{-1}\right)=$ $65.81-(0.1847 \times \mathrm{bpm}){ }^{23}$ The Sit and Reach Test was performed to measure the flexibility of the lower back and hamstring muscles. ${ }^{24}$ The Abdominal Curl Test, an endurance strength test for abdominal muscles, was used to record the 
total number of curl-ups performed by each participant in 60 seconds. ${ }^{25}$ The Flamingo Balance Test assessed the ability to balance on a single-leg-standing position. The total number of times of loss of balance in 60 seconds was recorded. ${ }^{26}$ The Shuttle Test assessed anaerobic power, speed and agility. The total time taken to consecutively run 10 times the distance of $5 \mathrm{~m}$ between two markers was recorded. ${ }^{24}$

\section{Statistical analysis}

Analysis of variance (ANOVA) was performed to assess the homogeneity of the three groups in terms of age, gender,
BMI, weight, \%FM and FFM, measured at baseline. Repeated-measures ANOVA was performed to assess significant differences in the anthropometric measures, body composition and fitness parameters scores, among the three different time point evaluations (within factor named time: pre vs. intermediate vs. post evaluation), among the three groups (between factor named groups: MRT vs. HRT vs. AT) and for the interaction time $\times$ groups. The dependent variables analyzed are shown in Table 1 . Bonferroni post hoc test was used to assess mean differences where a significant $F$ was observed. The alpha test level for statistical significance

Table I Results obtained by the three groups in the pre, intermediate and post assessments

\begin{tabular}{|c|c|c|c|c|}
\hline & & MRT & HRT & AT \\
\hline & & Means (SD) & Means (SD) & Means (SD) \\
\hline \multicolumn{5}{|l|}{ Anthropometric measures } \\
\hline \multirow[t]{3}{*}{ Weight (kg) } & Pre & $69.18(18.72)$ & $68.51(13.15)$ & $68.32(14.05)$ \\
\hline & Intermediate & $70.04(16.70)$ & 70.47 ( 11.37$)$ & $68.50(13.62)$ \\
\hline & Post & $71.25(15.46)$ & 72.24 (11.39) & $70.00(11.42)$ \\
\hline \multirow[t]{3}{*}{ Height (m) } & Pre & $1.57(0.09)^{\mathrm{a}}$ & $1.56(0.08)^{\mathrm{a}}$ & $1.59(0.07)$ \\
\hline & Intermediate & $1.59(0.10)$ & $1.58(0.07)^{\mathrm{a}}$ & $1.60(0.07)$ \\
\hline & Post & $\mathrm{I} .6 \mathrm{I}(0.07)^{\mathrm{b}}$ & $\mathrm{I} .60(0.05)^{\mathrm{b}, \mathrm{c}}$ & $1.62(0.06)$ \\
\hline \multirow[t]{3}{*}{ BMI $\left(\mathrm{kg} \cdot \mathrm{m}^{-2}\right)$} & Pre & $27.8 I(5.4 I)$ & $27.48(3.59)$ & $26.93(3.92)$ \\
\hline & Intermediate & $27.54(4.23)$ & $28.58(4.67)$ & $27.57(4.39)$ \\
\hline & Post & $26.96(3.85)$ & $27.84(1.48)$ & $26.45(3.44)$ \\
\hline \multirow[t]{3}{*}{ Waist circumference $(\mathrm{cm})$} & Pre & $84.20(9.67)$ & $88.21(9.61)$ & $88.54(10.89)$ \\
\hline & Intermediate & $83.35(8.97)$ & $83.75(9.1)$ & $88.38(10.85)$ \\
\hline & Post & $83.23(7.97)$ & $83.64(6.39)$ & $85.40(11.97)$ \\
\hline \multirow[t]{3}{*}{ WHR } & Pre & $0.81(0.05)$ & $0.88(0.09)$ & $0.89(0.07)$ \\
\hline & Intermediate & $0.84(0.10)$ & $0.86(0.08)$ & $0.89(0.07)$ \\
\hline & Post & $0.84(0.09)$ & $0.87(0.09)$ & $0.87(0.12)$ \\
\hline \multicolumn{5}{|l|}{ Body composition } \\
\hline \multirow[t]{3}{*}{$\% F M$} & Pre & $34.07(6.70)^{\mathrm{a}, \mathrm{c}}$ & $33.33(7.29)^{\mathrm{a}, \mathrm{c}}$ & $32.92(6.42)$ \\
\hline & Intermediate & $31.11(5.00)^{b}$ & $30.92(6.04)^{b}$ & $33.50(6.23)$ \\
\hline & Post & $29.41(3.52)^{b}$ & $30.28(3.39)^{b}$ & $32.56(5.4 I)$ \\
\hline \multirow[t]{3}{*}{$\mathrm{FFM}(\mathrm{kg})$} & Pre & $46.80(10.05)$ & $45.31(8.55)^{a}$ & $45.78(10.59)$ \\
\hline & Intermediate & $45.77(I I .8 I)^{\mathrm{a}}$ & $48.40(8.01)$ & $46.45(10.6)$ \\
\hline & Post & $49.92(8.16)^{c}$ & $49.83(5.87)^{b}$ & $48.66(8.61)$ \\
\hline \multicolumn{5}{|l|}{ Physical fitness test } \\
\hline Flamingo Balance Test & Pre & $7.83(2.98)^{\mathrm{a}, \mathrm{c}}$ & $7.50(2.7 I)^{a, c}$ & $7.25(2.77)$ \\
\hline \multirow[t]{2}{*}{ (seconds) } & Intermediate & $3.23(3.19)^{\mathrm{b}, *}$ & $3.30(2.45)^{\mathrm{b}, *}$ & $8.08(2.78)^{\mathrm{a}}$ \\
\hline & Post & $2.36(2.98)^{\mathrm{b}, *}$ & $3.00(2.42)^{\mathrm{b}, *}$ & $5.70(2.63)^{c}$ \\
\hline \multirow[t]{3}{*}{ Shuttle Test (seconds) } & Pre & $25.36(2.49)^{\mathrm{a}}$ & $25.05(2.53)^{\mathrm{a}}$ & $26.17(1.66)$ \\
\hline & Intermediate & $24.06(3.46)^{*}$ & $23.10(1.46)^{*}$ & $27.41(2.54)^{\mathrm{a}}$ \\
\hline & Post & $22.44(2.57)^{\mathrm{b}, *}$ & $22.43(1.00)^{\mathrm{b}, *}$ & $24.9(1.08)^{c}$ \\
\hline Abdominal Curl Test & Pre & I $8.88(4.9)^{\mathrm{a}}$ & $19.79(8.86)^{a}$ & $19.33(5.26)$ \\
\hline \multirow[t]{2}{*}{ (repetitions in 60 seconds) } & Intermediate & $19.85(2.95)^{\mathrm{a}}$ & $20.00(2.5 \mathrm{I})^{\mathrm{a}}$ & $19.83(5.75)$ \\
\hline & Post & $23.45(3.59)^{\mathrm{b}, \mathrm{c}}$ & $25.86(2.89)^{\mathrm{b}, \mathrm{c}}$ & $21.80(5.09)$ \\
\hline \multirow[t]{3}{*}{ Sit and Reach Test $(\mathrm{cm})$} & Pre & $\mid 2.92(5.9 \mid)$ & $10.86(4.3 \mid)$ & $12.00(4.66)$ \\
\hline & Intermediate & $15.60(5.34)$ & $14.57(3.29)$ & $12.33(3.95)$ \\
\hline & Post & $16.14(6.10)$ & $14.79(2.87)$ & $12.65(3.54)$ \\
\hline \multirow{3}{*}{$\mathrm{VO}_{2} \max \left(\mathrm{mL} \mathrm{O}{ }_{2} \cdot \mathrm{kg}^{-1} \cdot \mathrm{min}^{-1}\right)$} & Pre & $31.40(3.63)^{\mathrm{a}}$ & $30.21(3.02)^{\mathrm{a}}$ & $31.50(2.15)$ \\
\hline & Intermediate & $32.47(3.43)$ & $31.36(3.39)$ & $31.53(1.98)$ \\
\hline & Post & $34.23(3.20)^{\mathrm{b}, *}$ & $33.36(3.27)^{\mathrm{b}, *}$ & $32.52(2.54)^{\mathrm{b}}$ \\
\hline
\end{tabular}

Notes: a Significant $p$-value in the comparison vs. post score. ${ }^{b}$ Significant $p$-value in the comparison vs. pre score. 'Significant $p$-value in the comparison vs. intermediate score. *Significant $p$-value in the comparison vs. AT.

Abbreviations: MRT, moderate-resistance training; HRT, high-resistance training; AT, aerobic training; SD, standard deviation; BMI, body mass index; WHR, waist-hip ratio; \%FM, percentage of fat mass; FFM, free fat mass. 
was set at 0.05 . Effect size was calculated as $\eta^{2}$. For all the statistical analyses, the SPSS statistical software package was used (version 20.0; IBM).

For the analysis of the follow-up data, the number of POS-CO and NEG-CO of each group was calculated, and a chi-squared $3 \times 2$ (three groups $\times$ two categories) was performed to find significant differences among the three groups in physical activities participation after the end of the intervention (chi-squared $2 \times 2$ was instead used for paired comparisons among the groups). The variations $(\Delta s)$ between the follow-up scores and the phase I and phase II evaluation scores were calculated. The $\Delta$ was calculated for each group (MRT, HRT and AT) and for each category (POS-CO and NEG-CO) for the following four variables: weight, BMI, \%FM and FFM. This analysis aimed to assess the long-term modification of the three-group participants in comparison with their starting condition (baseline evaluation) and the condition at the end of the intervention (phase II evaluation), depending on whether they continued to perform physical activity.

The alpha test level for statistical significance was set at 0.05 .

\section{Results}

The sample characteristics are shown in Table 2.

The repeated-measures ANOVA showed significant differences among the time for height $\left(F_{2,76}=21.836\right.$; $p<$ $\left.0.001 ; \eta^{2}=0.365\right), \% \mathrm{FM}\left(F_{2,76}=5.843 ; p=0.004 ; \eta^{2}=\right.$ $0.133), \operatorname{FFM}\left(F_{2,76}=6.254 ; p=0.003 ; \eta^{2}=0.141\right)$, Flamingo Balance Test $\left(F_{2,76}=32.597 ; p<0.001 ; \eta^{2}=0.462\right)$, Sit and Reach Test $\left(F_{2,76}=9.678 ; p<0.001 ; \eta^{2}=0.203\right)$, Shuttle Test $\left(F_{2,76}=7.988 ; p=0.001 ; \eta^{2}=0.174\right)$, Abdominal Curl Test $\left(F_{2,76}=8.477 ; p<0.001 ; \eta^{2}=0.182\right)$, and $\mathrm{VO}_{2} \max$ $\left(F_{2,76}=32.692 ; p<0.001 ; \eta^{2}=0.462\right)$. Significant differences among the groups were found for Flamingo Balance Test $\left(F_{2,38}=72.555 ; p=0.013 ; \eta^{2}=0.205\right)$, Shuttle Test $\left(F_{2,38}=11.637 ; p<0.001 ; \eta^{2}=0.380\right)$, and $\mathrm{VO}_{2} \max$ $\left(F_{2,38}=4.264 ; p=0.021 ; \eta^{2}=0.183\right)$. Finally, significant differences in the interaction time $\times$ group were found for $\% \mathrm{FM}\left(F_{4,76}=3.333 ; p=0.014 ; \eta^{2}=0.149\right)$ and Flamingo
Balance Test $\left(F_{4,76}=8.331 ; p<0.001 ; \eta^{2}=0.304\right)$. The scores obtained by each group at baseline, phase I and phase II assessments and post hoc statistical analysis results are reported in Table 1.

From baseline to 16 weeks (phase I), the rate of adherence to exercise program was an average of $94 \%$ (each participant performed at least 40 sessions of the expected 48, with an average of 45 sessions performed) in both MRT and HRT groups with no significant differences between groups, whereas in the AT group, it was $83 \%$. From the 16 th to the 22 nd week (phase II), the rate of adherence was $>98 \%$ for the two groups (at least 16 sessions performed of the expected 18 ), and in the AT group, it was $78 \%$.

The results of the chi-squared test showed significant differences among the three groups relative to participation in physical activity after the end of the intervention (chi ${ }^{2}$ value $=6.73 ; p=0.035$ ). The comparison in pair showed that MRT $\left(\mathrm{chi}^{2}\right.$ value $\left.=4.64 ; p=0.031\right)$ and HRT $\left(\mathrm{chi}^{2}\right.$ value $=$ 5.57; $p=0.018$ ) groups had a significantly high number of subjects who continued the physical activity in comparison with the AT group. The $\% \Delta$ of the follow-up scores compared with baseline and phase II assessment scores are reported in Table 3.

\section{Discussion}

This study proposed a new combination of training protocols for overweight adolescents, designed with a first phase of RT (MRT or HRT) that prepares the subjects to the successive second phase of AP, and compared it to AP performed in both the first and the second phase.

AP is the traditional intervention program to shift the balance between the intake and expenditure of energy, decreasing waist circumferences and consequently abdominal fat accumulation, which is associated with a great risk of developing overweight pathologies. ${ }^{27} \mathrm{AP}$ represents the widely used type of physical training for correction of excessive body mass by increasing the energy expenditure. ${ }^{28}$ The problem is that this kind of training does not have a great sustainability for overweight subjects, ${ }^{8}$ given the higher physical and physiological demands, and does not provide the best chance

Table 2 Sample description

\begin{tabular}{llllll}
\hline Group & Age (years) & Weight $\mathbf{( k g )}$ & Height $(\mathbf{m})$ & BMI $\left(\mathbf{k g} \cdot \mathbf{m}^{-2}\right)$ & Fat mass $(\%)$ \\
\hline MRT & $12.73 \pm 0.70$ & $69.18 \pm 18.72$ & $1.57 \pm 0.09$ & $27.81 \pm 5.41$ & $34.07 \pm 6.70$ \\
HRT & $12.21 \pm 0.43$ & $68.51 \pm 13.15$ & $1.56 \pm 0.08$ & $27.48 \pm 3.59$ & $33.33 \pm 7.29$ \\
AT & $12.67 \pm 0.65$ & $68.32 \pm 14.05$ & $1.59 \pm 0.07$ & $26.93 \pm 3.92$ & $32.92 \pm 6.42$ \\
Total & $12.54 \pm 0.64$ & $68.59 \pm 15.45$ & $1.57 \pm 0.08$ & $27.25 \pm 4.51$ & $33.48 \pm 6.67$ \\
\hline
\end{tabular}

Note: The three groups were homogeneous at baseline.

Abbreviations: BMI, body mass index; MRT, moderate-resistance training; HRT, high-resistance training; AT, aerobic training. 
Table 3 Differences between follow-up scores compared to pre and post scores

\begin{tabular}{|c|c|c|c|c|c|c|}
\hline \multirow{2}{*}{$\begin{array}{l}\text { Group } \\
\text { Category }\end{array}$} & \multicolumn{2}{|l|}{ MRT } & \multicolumn{2}{|l|}{ HRT } & \multicolumn{2}{|l|}{ AT } \\
\hline & POS-CON & NEG-CON & POS-CON & NEG-CON & POS-CON & NEG-CON \\
\hline Numerosity & 10 & 5 & 10 & 4 & 3 & 9 \\
\hline Significance & \multicolumn{2}{|c|}{$\mathrm{Chi}^{2}=4.64 ; p=0.031^{*}$} & \multicolumn{2}{|c|}{$C h i^{2}=5.57 ; p=0.018^{*}$} & & \\
\hline \multicolumn{7}{|c|}{ Comparison with the pre assessments } \\
\hline Weight (kg) & -5.4 & +3.1 & $-6,0$ & +2.9 & -2.4 & +3.1 \\
\hline BMI $\left(\mathrm{kg} \cdot \mathrm{m}^{-2}\right)$ & -2.6 & +2.8 & -1.9 & +1.1 & -1.3 & +1.6 \\
\hline$\% F M$ & -4.8 & +1.8 & -5.4 & +2.2 & -2.3 & +0.5 \\
\hline $\mathrm{FFM}(\mathrm{kg})$ & +4.7 & -0.8 & +5.1 & -1.7 & +1.2 & -0.6 \\
\hline \multicolumn{7}{|c|}{ Comparison with the post assessments } \\
\hline Weight (kg) & -5.2 & +1.9 & -6.3 & +1.7 & $-2,9$ & +2.2 \\
\hline BMI $\left(\mathrm{kg} \cdot \mathrm{m}^{-2}\right)$ & -1.5 & +3.3 & -1.7 & +2.6 & -1.1 & +2.1 \\
\hline$\% F M$ & -0.7 & +6.3 & -2.7 & +5.6 & -2.2 & +2.2 \\
\hline $\mathrm{FFM}(\mathrm{kg})$ & +1.9 & -3.2 & +1.1 & -4.4 & +0.4 & -3.3 \\
\hline
\end{tabular}

Notes: POS-CON (positive continuer): participants who continued to perform physical activities after the end of the study; NEG-CON (negative continuer): participants who did not continue to perform physical activities after the end of the study. *Statistically significant in comparison with AT.

Abbreviations: MRT, moderate-resistance training; HRT, high-resistance training; AT, aerobic training; BMI, body mass index; \%FM, percentage of fat mass; FFM, free fat mass.

to compete with slimmer peers. Moreover, overweight teenagers, performing prolonged periods of aerobic exercise in which most overweight teens "fail", perceived themselves to be under constant scrutiny from their peers and ridiculed by them. ${ }^{29}$ Conversely, RT is an exercise modality in which overweight adolescents can excel relatively to their peers, given their larger FFM. ${ }^{30}$ Since this kind of training is enjoyable and satisfying, it can influence the adoption of active behaviors, ${ }^{26}$ considering that physical inactivity is strongly related to adolescent obesity. ${ }^{31}$ In the first part of this study, we tried to establish a dose-response relationship comparing moderate- vs. high-intensity RT protocols, and consequently to assess the most effective RT intensity. Our results showed the same effect of both RT protocols on body composition and fitness parameters. Preview studies showed that high-intensity progressive training decreases adiposity and improves the related metabolic outcomes, more than low- and moderate-intensity trainings. In the present study, the differences in intensity between the two RT protocols were not very relevant (9-RM vs. 13-RM) because more vigorous intensity would have not been suitable for this population. After phase II, the participants of both RT groups showed significant improvements in body composition (\%FM and FFM) and in physical fitness tests (Flamingo Balance Test, Shuttle Test and $\mathrm{VO}_{2}$ max), compared to baseline and AT groups, with no differences between them (Table 1). AT group showed a significant improvement only in the Flamingo Balance Test, Shuttle Test and $\mathrm{VO}_{2}$ max after phase II. The significant improvements in FFM and related skills, such as balance and speed (assessed by Flamingo Balance Test and Shuttle Test, respectively), after phase II, confirmed the utility of performing RT before AP. The nonsignificant changes in anthropometric measures may be due to the intervention without a dietary control. However, dietary restriction has been shown to cause FFM loss that could negatively counteract the RT gains..$^{32,33}$ Moreover, dietary intervention in addition to physical activity protocol would have been a confounding variable in the present study. ${ }^{27,34,35}$ Previous findings indicated that the combined training (RT plus AP) was the best choice for obese and overweight adolescent physical activity treatment. ${ }^{27}$ However, the combination of the two activities may be heavy, too long and unpleasant, accelerating the risk of incurring a decrease in enjoyment and endangering the lifelong commitment to physical activity necessary to prevent weight regain. ${ }^{7}$ Overweight participants, choosing activities as RT which promote vigorous exertion and physical changes, may improve their self-confidence and self-efficacy in carrying out the successive AP protocol. The level of satisfaction of adolescents with the intervention program was assessed by the adherence to the physical activity programs and by the number of dropped-out subjects. The dropped-out subjects in this study were all girls, and it is well known that overweight females are more acutely sensitive to any implied criticism relating to their body, bodily performance or social practices. Nevertheless, it has to be remarked that almost all the dropped-out subjects belonged to the AT group. ${ }^{36}$

The adherence of the two experimental groups was significantly higher than that of AT, and more participants in the experimental groups passed from the first phase to the second phase. Participants in MRT and HRT groups, in fact, showed significantly more adherence and compliance in the following AP than those in AT. The subjects who discontinued 
physical activity after all the three intervention protocols showed a weight increase especially in terms of \% FM and loss of FFM. Those who carried on the physical activity after the intervention continued to lose weight, maintaining the FFM level achieved.

Therefore, this program of training may prepare participants to be more available to continue the AP and to assume a more active lifestyle as it was demonstrated by follow-up results.

\section{Conclusion}

RT followed by AP can be an effective treatment option for obese youth. This is the first study that proposes an intervention based on different exercise protocols in sequence for overweight population. Overweight adolescents, who want to maximize the effect of the physical activity, should prepare themselves through RT before performing AP exercises, which assures them safe physical and health benefits. ${ }^{37,38}$

The positive effect of the programs proposed in this study was the global maintenance of training effects after intervention, ${ }^{39}$ motivating the adolescents to adopt an active lifestyle. ${ }^{11}$ MRT or HRT performed before AP, in fact, increased longterm exercise adherence, compared to AP alone. ${ }^{8}$ These findings represent an advancement in the theory-based physical activity intervention for overweight adolescents.

\section{Disclosure}

The authors report no conflicts of interest in this work.

\section{References}

1. Lloyd RS, Faigenbaum AD, Stone MH, et al. Position statement on youth resistance training: the 2014 International Consensus. Br J Sports Med. 2014;48(7):498-505.

2. Faigenbaum AD, Myer GD. Resistance training among young athletes: safety, efficacy and injury prevention effects. Br J Sports Med. 2010;44(1):56-63.

3. Docherty D, Wenger HA, Collis ML. The effects of resistance training on aerobic and anaerobic power of young boys. Med Sci Sports Exerc. 1987;19(4):389-392.

4. Benson AC, Torode ME, Fiatarone Singh MA. The effect of highintensity progressive resistance training on adiposity in children: a randomized controlled trial. Int J Obes (Lond). 2008;32(6):1016-1027.

5. Durrer C, Robinson E, Wan Z, et al. Differential impact of acute highintensity exercise on circulating endothelial microparticles and insulin resistance between overweight/obese males and females. PLoS One. 2015;10(2):e0115860.

6. Donnelly JE, Blair SN, Jakicic JM, Manore MM, Rankin JW, Smith BK; American College of Sports Medicine. American College of Sports Medicine Position Stand. Appropriate physical activity intervention strategies for weight loss and prevention of weight regain for adults. Med Sci Sports Exerc. 2009;41(2):459-471.

7. Thiel C, Vogt L, Claussnitzer G, Banzer W. Energy cost of youth obesity exercise modes. Int J Sports Med. 2011;32(2):142-146.

8. Gillis LJ, Kennedy LC, Bar-Or O. Overweight children reduce their activity levels earlier in life than healthy weight children. Clin J Sport Med. 2006;16(1):51-55.
9. Ekkekakis P. Let them roam free? Physiological and psychological evidence for the potential of self-selected exercise intensity in public health. Sports Med. 2009;39(10):857-888.

10. Faigenbaum AD, Kraemer WJ, Blimkie CJ, et al. Youth resistance training: updated position statement paper from the National Strength and Conditioning Association. J Strength Cond Res. 2009;23(5 Suppl):S60-S79.

11. Haines J, Kleinman KP, Rifas-Shiman SL, Field AE, Austin SB. Examination of shared risk and protective factors for overweight and disordered eating among adolescents. Arch Pediatr Adolesc Med. 2010;164(4):336-343.

12. Patton GC, Coffey C, Carlin JB, et al. Overweight and obesity between adolescence and young adulthood: a 10-year prospective cohort study. $J$ Adolesc Health. 2011;48(3):275-280.

13. Wilson AJ, Jung ME, Cramp A, Simatovic J, Prapavessis H, Clarson C. Effects of a group-based exercise and self-regulatory intervention on obese adolescents' physical activity, social cognitions, body composition and strength: a randomized feasibility study. J Health Psychol. 2012;17(8):1223-1237.

14. Shah NR, Braverman ER. Measuring adiposity in patients: the utility of body mass index (BMI), percent body fat, and leptin. PLoS One. 2012;7(4):e33308.

15. Ratcliff MB, Jenkins TM, Reiter-Purtill J, Noll JG, Zeller MH. Risktaking behaviors of adolescents with extreme obesity: normative or not? Pediatrics. 2011;127(5):827-834.

16. Brzycki M. Strength testing - predicting a one-rep max from reps-tofatigue. J Phys Educ Recreat Dance. 1993;64(1):88-90.

17. Moreno LA, Pineda I, Rodríguez G, Fleta J, Sarría A, Bueno M. Waist circumference for the screening of the metabolic syndrome in children. Acta Paediatr. 2002;91(12):1307-1312.

18. Johnston JL, Leong MS, Checkland EG, Zuberbuhler PC, Conger PR, Quinney HA. Body fat assessed from body density and estimated from skinfold thickness in normal children and children with cystic fibrosis. Am J Clin Nutr. 1988;48(6):1362-1366.

19. Weststrate JA, Deurenberg P. Body composition in children: proposal for a method for calculating body fat percentage from total body density or skinfold-thickness measurements. Am J Clin Nutr. 1989;50(5):1104-1115.

20. Rodríguez G, Moreno LA, Blay MG, et al; AVENA-Zaragoza Study Group. Body fat measurement in adolescents: comparison of skinfold thickness equations with dual-energy X-ray absorptiometry. Eur J Clin Nutr. 2005;59(10):1158-1166.

21. Council of Europe, Committee for the Development of Sport. EUROFIT. European Test of Physical Fitness. Rome: Council of Europe, Committee for the Development of Sport; 1988.

22. Chatterjee $S$, Chatterjee P, Bandyopadhyay A. Enumeration of validity for predicted $\mathrm{VO}_{2}$ max by Queen's College Step Test in Bengalee boys. Ind J Physiol Allied Sci. 2001;55(3):123-127.

23. McArdle WD, Katch FI, Pechar GS, Jacobson L, Ruck S. Reliability and interrelationships between maximal oxygen intake, physical work capacity and step test scores in college women. Med Sci Sports. 1972;4(4):182-186.

24. Vanhees L, Lefevre J, Philippaerts R, et al. How to assess physical activity? How to assess physical fitness? Eur J Cardiovasc Prev Rehabil. 2005;12(2):102-114.

25. YMCA of the USA. YMCA Fitness Testing and Assessment Manual. Golding LA, editor. Champaign, IL: Human Kinetics; 2000.

26. Rothman AJ. Toward a theory-based analysis of behavioral maintenance. Health Psychol. 2000;19(1 Suppl):64-69.

27. Sigal RJ, Alberga AS, Goldfield GS, et al. Effects of aerobic training, resistance training, or both on percentage body fat and cardiometabolic risk markers in obese adolescents: the healthy eating aerobic and resistance training in youth randomized clinical trial. JAMA Pediatr. 2014;168(11):1006-1014.

28. Sawczyn S, Mishchenko V, Moska W, et al. Strength and aerobic training in overweight females in Gdansk, Poland. Open Med. 2015;10:152-162.

29. Faigenbaum AD. Strength training for overweight teenagers. Strength Cond J. 2002;24(5):67-68. 
30. Stankov I, Olds T, Cargo M. Overweight and obese adolescents: what turns them off physical activity? Int J Behav Nutr Phys Act. 2012;9:53.

31. Page A, Cooper AR, Stamatakis E, et al. Physical activity patterns in nonobese and obese children assessed using minute-by-minute accelerometry. Int J Obes (Lond). 2005;29(9):1070-1076.

32. Lee S, Bacha F, Hannon T, Kuk JL, Boesch C, Arslanian S. Effects of aerobic versus resistance exercise without caloric restriction on abdominal fat, intrahepatic lipid, and insulin sensitivity in obese adolescent boys: a randomized, controlled trial. Diabetes. 2012;61(11):2787-2795.

33. Di Cagno A, Marchetti M, Battaglia C, et al. Is menstrual delay a serious problem for elite rhythmic gymnasts? J Sports Med Phys Fitness. 2012;52(6):647-653.

34. Hamasaki H, Kawashima Y, Tamada Y, et al. Associations of lowintensity resistance training with body composition and lipid profile in obese patients with type 2 diabetes. PLoS One. 2015;10(7): e0132959.
35. Schranz N, Tomkinson G, Olds T. What is the effect of resistance training on the strength, body composition and psychosocial status of overweight and obese children and adolescents? A systematic review and meta-analysis. Sports Med. 2013;43(9):893-907.

36. Curtis, P. The experiences of young people with obesity in secondary school: some implications for the healthy school agenda. Health Soc Care Community. 2008;16(4):410-418.

37. di Cagno A, Battaglia C, Giombini A, et al. Time of day-effects on motor coordination and reactive strength in elite athletes and untrained adolescents. J Sports Sci Med. 2013;12(1):182-189.

38. di Cagno A, Fiorilli G, Iuliano E, et al. Time-of-day effects on static and dynamic balance in elite junior athletes and untrained adolescents. Int J Sports Sci Coach. 2014;9(4):615-625.

39. Piazza M, Battaglia C, Fiorilli G, et al. Effects of resistance training on jumping performance in pre-adolescent rhythmic gymnasts: a randomized controlled study. Ital J Anat Embryol. 2014;119(1):10-19.
Diabetes, Metabolic Syndrome and Obesity: Targets and Therapy is an international, peer-reviewed open-access journal committed to the rapid publication of the latest laboratory and clinical findings in the fields of diabetes, metabolic syndrome and obesity research. Original research, review, case reports, hypothesis formation, expert

\section{Dovepress}

opinion and commentaries are all considered for publication. The manuscript management system is completely online and includes a very quick and fair peer-review system, which is all easy to use. Visit http://www.dovepress.com/testimonials.php to read real quotes from published authors.

Submit your manuscript here: https://www.dovepress.com/diabetes-metabolic-syndrome-and-obesity-targets-and-therapy-journal 